\title{
Improved door-to-balloon time for primary percutaneous coronary intervention for patients conveyed via emergency ambulance service
}

\author{
Zhenghong Liu ${ }^{1}$ MBBS, Mian Jie $\underline{\operatorname{Lim}}{ }^{2}{ }_{M B B S}$, Pin Pin Pek ${ }^{1,3}{ }_{M P H}$, Aaron Sung Lung Wong ${ }^{4}{ }_{M R C P(U K)}$,
} Kenneth Boon Kiat $\underline{\operatorname{Tan}}^{1}{ }^{M M e d}(E M)$, Khung Keong Yeo ${ }^{4}{ }_{A B I M}$, Marcus Eng Hock Ong ${ }^{1,4}$ MPH

\begin{abstract}
Introduction: Early reperfusion of ST-segment elevation myocardial infarction (STEMI) results in better outcomes. Interventions that have resulted in shorter door-to-balloon (DTB) time include prehospital cardiovascular laboratory activation and prehospital electrocardiogram (ECG) transmission, which are only available for patients who arrive via emergency ambulances. We assessed the impact of mode of transport on DTB time in a single tertiary institution and evaluated the factors that affected various components of DTB time.

Methods: We conducted a retrospective cohort study using registry data of patients diagnosed with STEMI in the emergency department (ED) who underwent primary percutaneous coronary intervention. We compared patients who arrived by emergency ambulances with those who came via their own transport. The primary study end point was DTB, defined as the earliest time a patient arrived in the ED to balloon inflation. As deidentified data was used, ethics review was waived.

Results: A total of 321 patients were included for analysis after excluding 7 with missing data. The mean age was $61.4 \pm 11.4$ years old with 49 (15.3\%) females. Ninety-nine $(30.8 \%)$ patients arrived by emergency ambulance. The median DTB time was shorter for patients arriving by ambulance versus own transport (52min, interquartile range [IQR] 45-61 vs 67min, IQR 59-74; $P<0.001$ ), with shorter door-to-ECG and door-to-activation time.

Conclusion: Arrival via emergency ambulance was associated with a decreased DTB for STEMI patients compared to arriving via own transport. There is a need for public education to increase the usage of emergency ambulances for suspected heart attacks to improve outcomes.
\end{abstract}

Ann Acad Med Singap 2021;50:671-8

Keywords: Cardiovascular lab activation, door-to-balloon time, emergency ambulance, primary PCI, STEMI

\section{INTRODUCTION}

Early reperfusion of ST-segment elevation myocardial infarction (STEMI) has been shown to result in better outcomes, ${ }^{1-3}$ and guidelines for treatment of STEMI recommend a rapid and coordinated response. ${ }^{4}$ The American Heart Association guidelines released in 2014 recommend a door-to-balloon (DTB) time of less than 90 minutes, ${ }^{5}$ and timings of less than 60 minutes have been associated with better outcomes. ${ }^{6}$

In Singapore, patients presenting to the emergency departments (ED) of our public hospitals with acute STEMI are usually offered emergency percutaneous coronary intervention (PCI) as first-line treatment. In line with international recommendations, the national target for DTB time is 90 minutes. This target is met by public hospitals more than $90 \%$ of the time. ${ }^{7}$

Worldwide, interventions that have resulted in shorter DTB time include direct emergency physician activation of cardiovascular laboratory (CVL), prehospital CVL activation and prehospital electrocardiogram (ECG) transmission. ${ }^{8-10}$ As these interventions are only available to patients being conveyed by emergency ambulances, it is recommended that patients should call an emergency ambulance when experiencing symptoms such as severe

\footnotetext{
${ }^{1}$ Department of Emergency Medicine, Singapore General Hospital, Singapore

${ }^{2}$ SingHealth Emergency Medicine Residency Programme, SingHealth, Singapore

${ }^{3}$ Prehospital and Emergency Research Centre, Health Services and Systems Research, Duke-NUS Medical School, Singapore

${ }^{4}$ Department of Cardiology, National Heart Centre Singapore, Singapore

Correspondence: Dr Zhenghong Liu, Department of Emergency Medicine, Singapore General Hospital, Outram Road, Singapore 169608.

Email: liu.zhenghong@singhealth.com.sg
} 


\section{CLINICAL IMPACT}

\section{What is New}

- This study shows that emergency ambulance usage is associated with shorter door-to-balloon (DTB) time largely due to shorter door-toelectrocardiogram timing.

- Other aspects that require attention include after-office-hours timing.

\section{Clinical Implications}

- The study supports the need to increase the awareness and usage of emergency ambulances when a heart attack is suspected.

- The findings can guide further investigations and quality improvement projects to reduce DTB time.

chest pain, especially when associated with sweating, breathlessness, nausea or vomiting. Despite this, some international studies report that only $60 \%$ of STEMI patients use an ambulance. ${ }^{11}$ In Singapore, a previous study found that only $50 \%$ percent of STEMI patients come to hospital by emergency ambulance. ${ }^{12}$

We aimed to study the impact of mode of arrival on DTB time in our institution, Singapore General Hospital. We hypothesise that usage of emergency ambulances will be associated with shorter DTB time compared to arriving via own transport. Subsequently, we aimed to analyse the impact of mode of transport on the various components of DTB time. Amongst patients conveyed by emergency ambulances, we further analysed the impact of hospital ED standby, ECG transmission and CVL activation on the various components of DTB time.

\section{METHODS}

\section{Study design}

We conducted a single-centre retrospective cohort study of all patients who underwent emergency PCI for STEMI from the ED from January 2018 to August 2019. We conducted a chart review combining emergency department records, cardiology department registry data, as well as an ongoing national myocardial infarction registry. Ethics review was waived by the SingHealth Centralised Institutional Review Board (2020/3011).

\section{Setting}

Singapore General Hospital is Singapore's largest tertiary hospital with 1,735 beds. The ED sees an annual volume of more than 125,000 patients, with emergency
PCI supported by the National Heart Centre Singapore, a specialist heart centre.

All patients presenting to the ED with a complaint that could represent an acute coronary syndrome are prioritised for an ECG. This is done in the ambulatory triage area for ambulatory patients and in the critical care area for non-ambulatory patients. All ECGs are immediately reviewed by a senior doctor, who would assess for the presence of STEMI.

Singapore's emergency medical services system is run by the Singapore Civil Defence Force (SCDF). The service is activated by a centralised 995 dispatching system, with the service handling 191,468 ambulance calls in 2019. ${ }^{13}$ SCDF's 60 ambulances are manned by specially trained paramedics, who are able to put an ED on standby for patients in whom they suspect a STEMI, and are also able to fax ECGs on route to the ED. On receipt of an ECG diagnostic of a STEMI, the emergency physician has the option to activate the CVL lab prior to the patient's arrival. ${ }^{9,12}$

\section{Data source and study population}

All patients who presented to the ED with STEMI and subsequently underwent emergency PCI were included for analysis. Patients with missing data on mode of arrival or DTB time were excluded from analysis.

We obtained the list of patients from the Primary PCI database that is maintained by the National Heart Centre Singapore. This database comprises patients who underwent primary PCI from the emergency department. It contains demographic data and a breakdown of the components of DTB time, including the time of arrival, time of ECG, time of CVL activation, time of arrival to the CVL lab, as well as time of perfusion. The database has exclusion criteria that exclude patients who suffered a cardiac arrest, had cardiogenic shock requiring resuscitation or showed a non-diagnostic ECG. As such, only patients with clear STEMI on ECG and did not require prolonged resuscitation in the ED were included in this cohort. Full exclusion criteria are available in Table S1 of Supplementary Materials (in the online version of this article.)

Data on mode of arrival were obtained from the ED records. The triage note written by nurses contains information on the mode of arrival. We also matched outcomes from the Singapore Myocardial Infarction Registry, ${ }^{14}$ which is a national registry that contains outcome data pertaining to our patients. We also accessed the SCDF ECG transmission records to identify patients for whom prehospital ECGs were transmitted. Data were extracted by 2 study team members, with disagreements resolved by consensus. 


\section{Study variables}

Our primary outcome was DTB time in minutes, defined as the earliest time the patient was registered in the ED to the time the balloon was inflated. We also examined inpatient mortality as well as the components of DTB time as secondary outcomes, such as door-to-ECG time, and door-to-CVL time. Components of DTB time were recorded by the ED staff and CVL staff, and extracted from the Primary PCI database.

The independent variable was mode of arrival, which was obtained from the ED patient records. Other independent variables included prehospital transmission of ECG as well as whether the ED was put on standby. These were obtained from the ED records and SCDF ECG transmission records.

\section{Data analysis}

We described patient characteristics using percentages for categorical data. Mean and standard deviation, or median and interquartile range (IQR) were used for normally and non-normally distributed continuous variables, respectively. Statistical comparisons for differences in baseline characteristics among patients arriving by emergency ambulance and own transport were performed using chi-square tests for categorical variables. T-test and Wilcoxon rank-sum test were used for normally and non-normally distributed data, respectively. DTB time and its components were described using median and IQR based on mode of transport.

Subsequently, we conducted multivariable linear regression with DTB time in minutes as the primary outcome. For the predictors, we included factors that have been reported to be associated with DTB time, including age, sex, presentation during working hours, and ambulance usage. ${ }^{15,16}$ Secondary outcomes include door-to-ECG time, door-to-activation time, activationto-lab time and lab-to-balloon time.

The primary and secondary endpoints (in minutes) were $\log$ transformed for use in regression models to maintain normality. Statistical output is therefore reported as percent change in time and 95\% confidence interval. All tests were 2-sided, with statistical significance predefined as a $P$ value $<0.05$.

All statistical analyses were performed using $R$ software version 4.0.2.

\section{RESULTS}

\section{Patient characteristics}

There were 328 patients in the cohort from January 2018 to August 2019. After excluding 7 patients due to missing data, 321 patients were included for analysis.
Table 1 lists the patients' characteristics. Median age was 61.2 years and $82.9 \%$ were men. In terms of ethnicity, $62.6 \%$ were Chinese, $13.1 \%$ were Malay and $13.4 \%$ were Indian. Compared to Singapore Census of Population 2020 data (Chinese 74.3\%, Malays 13.5\%, Indians $9.0 \%$ ), a larger proportion of our study cohort were Indians.

In total, $99(30.8 \%)$ patients arrived by emergency ambulance and $222(69.2 \%)$ arrived via own transport. Fig. 1 shows the study flow chart. Among the characteristics listed in Table 1, age, diabetes mellitus and smoking status was different between those arriving via emergency ambulance versus own transport.

\section{Association between DTB timings, inpatient mortality and mode of transport}

Fig. 2 shows that the median DTB time was shorter for patients arriving by emergency ambulance (52 min, IQR 45-61) than by own transport (67 min, IQR 59-74, $P<0.001)$. Further analysis showed that arrival via emergency ambulance was also associated with shorter median door-to-ECG time (11 min, IQR 5-15 vs $1 \mathrm{~min}$, IQR $0-3 ; P<0.001)$ and door-to-activation (18 $\mathrm{min}$, IQR $12-25$ vs $2 \mathrm{~min}$, IQR $0.10-7.50 ; P<0.001)$ (Table 2). There was no difference in inpatient mortality among patients arriving by emergency ambulance vs own transport $(6.1 \%$ vs $2.7 \%, P=0.252)$

\section{DTB timings and prehospital measures}

Table 3 shows median DTB time of patients based on whether they had prehospital ED standby, ECG transmission and CVL activation. Patients arriving via emergency ambulance without prehospital standby had a median DTB time of more than 60 minutes. With each additional measure (standby, prehospital ECG transmission and prehospital CVL activation), there was a trend towards shorter DTB times.

\section{Other factors affecting various components of DTB time}

Table 4 shows the results of multivariate adjustment for potential confounders (age, sex, office hours arrival). Usage of emergency ambulance remained significantly associated with shorter DTB time (18.5\% shorter, 95\% CI $-22.3 \%$ to $-14.5 \%, P<0.001)$, largely attributable to decreased door-to-ECG $(87.7 \%$ shorter, $95 \%$ CI $-91.7 \%$ to $-81.8 \%, P<0.001)$ and door-to-CVL activation times $(90.6 \%$ shorter, $95 \%$ CI $-93.6 \%$ to $-86.2 \%, P<0.001)$. Presentation during office hours was also significantly associated with shorter DTB time $(8.5 \%$ shorter, $95 \% \mathrm{CI}-12.5 \%$ to $-4.3 \%, P<0.001)$, largely attributable to decreased time from arrival 


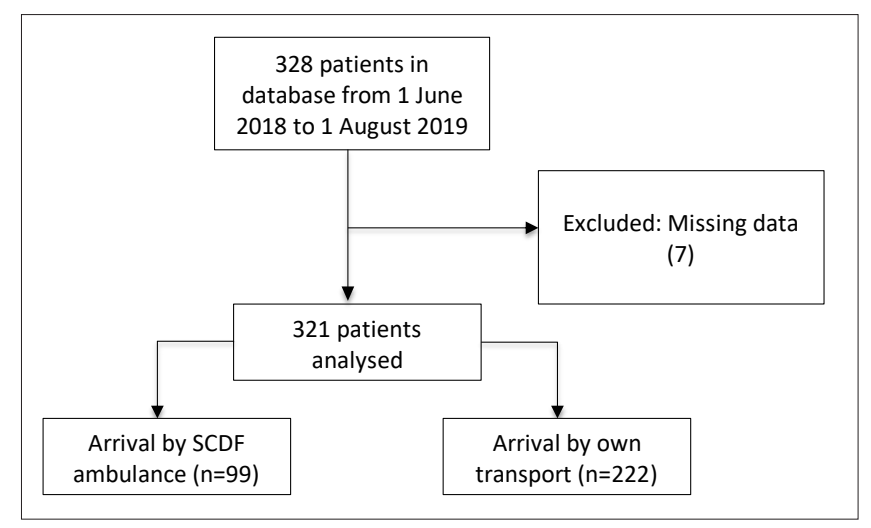

Fig. 1. Study flow chart.

SCDF: Singapore Civil Defence Force at CVL lab to balloon inflation $(21.1 \%$ shorter, $95 \%$ CI $-28.3 \%$ to $-13.2 \%, P<0.001$ )

\section{DISCUSSION}

\section{Emergency ambulance usage and DTB time}

In this study, we found that arrival via emergency ambulance was associated with DTB times roughly 15 minutes shorter than arriving via other modes of transport.

Prior studies have found that prehospital ECG transmission and CVL activation reduces DTB time. A nationwide, before- after-study in Singapore, showed a reduction of DTB time of 24 minutes after

Table 1. Characteristics of patients

\begin{tabular}{|c|c|c|c|}
\hline Characteristics & $\begin{array}{c}\text { Own transport }(\mathrm{n}=\mathbf{2 2 2}) \\
\text { No. }(\%)\end{array}$ & $\begin{array}{c}\text { Emergency ambulance }(\mathrm{n}=99) \\
\text { No. }(\%)\end{array}$ & $P$ value \\
\hline Man & $182(82.0)$ & $90(90.9)$ & 0.059 \\
\hline Age, mean (SD), years & $62.47(11.01)$ & $58.98(11.85)$ & 0.011 \\
\hline Race & & & 0.085 \\
\hline Chinese & $147(66.2)$ & $54(54.5)$ & \\
\hline Malay & $26(11.7)$ & $16(16.2)$ & \\
\hline Indian & $31(14.0)$ & $12(12.1)$ & \\
\hline Others & $18(8.3)$ & $17(17.1)$ & \\
\hline Office-hours presentation & $81(36.5)$ & $45(45.5)$ & 0.163 \\
\hline Day of the week & & & 0.152 \\
\hline Monday & $31(14.0)$ & $10(10.1)$ & \\
\hline Tuesday & $35(15.8)$ & $16(16.2)$ & \\
\hline Wednesday & $30(13.5)$ & $13(13.1)$ & \\
\hline Thursday & $26(11.7)$ & $16(16.2)$ & \\
\hline Friday & $29(13.1)$ & $21(21.2)$ & \\
\hline Saturday & $37(16.7)$ & $7(7.1)$ & \\
\hline Sunday & $34(15.3)$ & $16(16.2)$ & \\
\hline Diabetes mellitus & $78(35.3)$ & $20(21.1)$ & 0.017 \\
\hline Hypertension & $123(55.7)$ & $46(48.4)$ & 0.289 \\
\hline Dyslipidaemia & $128(57.9)$ & $47(49.5)$ & 0.207 \\
\hline Current smoker & $72(32.6)$ & $43(45.3)$ & 0.043 \\
\hline Prior myocardial infarction & $23(10.4)$ & $8(8.4)$ & 0.735 \\
\hline Cerebrovascular disease & $10(4.5)$ & $3(3.2)$ & 0.761 \\
\hline Peripheral arterial disease & $5(2.3)$ & $1(1.1)$ & 0.672 \\
\hline Current dialysis & $4(1.8)$ & $1(1.1)$ & 1 \\
\hline
\end{tabular}

SD: standard deviation 


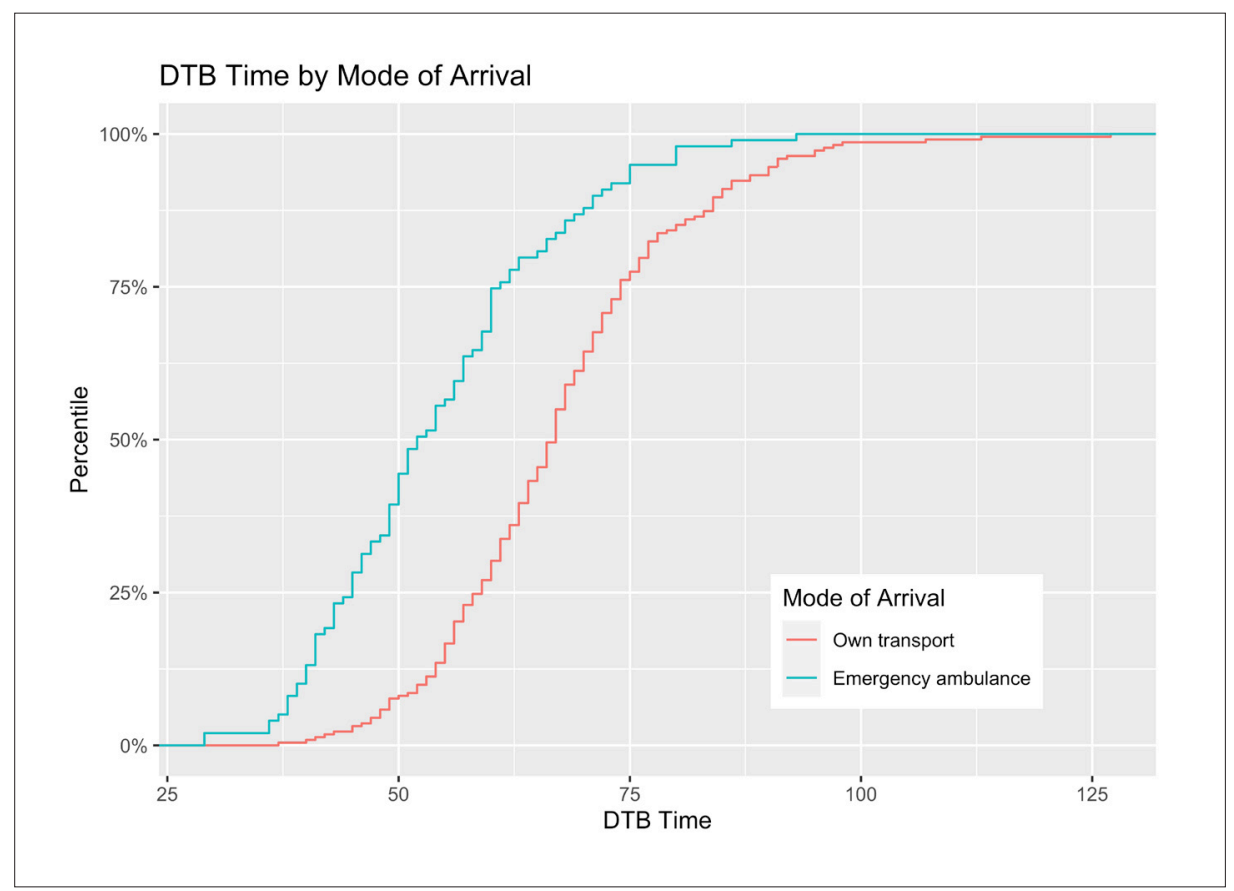

Fig. 2. DTB time by mode of arrival.

DTB: door-to-balloon

Table 2. Door-to-balloon (DTB) parameters and mortality by mode of arrival

\begin{tabular}{lccc}
\hline DTB parameters & Own transport (n=222) & Emergency ambulance (n=99) & $P$ value \\
\hline DTB time, median (IQR), min & $67.00(59.00-74.00)$ & $52.00(45.00-60.50)$ & $<0.001$ \\
\hline DTB time $<$ 60 min, no. (\%) & $67(30.2)$ & $74(74.7)$ & $<0.001$ \\
\hline DTB time $<90$ min, no. (\%) & $210(94.6)$ & $98(99.0)$ & 0.124 \\
\hline Deceased during stay, no. (\%) & $6(2.7)$ & $6(6.1)$ & 0.252 \\
\hline Door-to-ECG time, median (IQR), min & $11.00(5.00-15.00)$ & $1.00(0-3.00)$ & $<0.001$ \\
\hline Door-to-CVL activation time, median (IQR), min & $18.00(12.00-25.00)$ & $2.00(0.10-7.50)$ & $<0.001$ \\
\hline CVL activation to CVL arrival, median (IQR), min & $29.00(25.00-33.00)$ & $30.00(25.00-36.00)$ & 0.178 \\
\hline CVL arrival to balloon, median (IQR), min & $19.00(14.00-24.00)$ & $17.00(13.00-22.75)$ & 0.126 \\
\hline
\end{tabular}

CVL: cardiovascular laboratory; ECG: electrocardiogram; IQR: interquartile range

Table 3. Door-to-balloon (DTB) time for various prehospital factors

Arriving via own transport $(\mathrm{n}=222)$ $67.00(59.00-74.00)$

Arriving via SCDF but ED not put on standby $(n=7)$

Arrived via SCDF, ED put on standby but no ECG transmitted (n=37)

Arrived via SCDF, ED put on standby, ECG transmitted but no prehospital activation ( $\mathrm{n}=15)$

Arrived via SCDF, ED put on standby, ECG transmitted with prehospital activation $(n=40)$

ECG: electrocardiogram; ED: emergency department; IQR: interquartile range; SCDF: Singapore Civil Defence Force 
Table 4. Variables associated with change in components of door-to-balloon time after adjustment for confounders

\begin{tabular}{lccc}
\hline & Ambulance usage (95\% CI) & $P$ value \\
\hline Door-to-balloon time & $18.5 \%$ shorter (-22.3 to -14.5\%) & $<\mathbf{0 . 0 0 1}$ \\
\hline Door-to-ECG time & $87.7 \%$ shorter (-91.7 to -81.8\%) & $<\mathbf{0 . 0 0 1}$ \\
\hline Door-to-activation time & $90.6 \%$ shorter (-93.6 to -86.2\%) & $<\mathbf{0 . 0 0 1}$ \\
\hline Activation-to-lab time & $10.5 \%$ longer (-2.5 to $25.5 \%)$ & 0.12 \\
\hline Lab-to-balloon time & $6.2 \%$ shorter (-15.2 to 3.8\%) & 0.22 \\
\hline Door-to-balloon time & Office-hours presentation (95\% CI) & $\boldsymbol{P}$ value \\
\hline Door-to-ECG time & $8.5 \%$ shorter (-12.5 to -4.3\%) & $<\mathbf{0 . 0 0 1}$ \\
\hline Door-to-activation time & $1.5 \%$ longer (-29.6 to $46.2 \%)$ & 0.93 \\
\hline Activation-to-lab time & $1.4 \%$ shorter (-31.1 to $41.1 \%)$ & 0.94 \\
\hline Lab-to-balloon time & $9.7 \%$ shorter (-19.8 to $1.64 \%)$ & 0.09 \\
\hline
\end{tabular}

CI: confidence interval; ECG: electrocardiogram

$P$ values in bold are significant

implementation of out-of-hospital ECG recording and transmission. ${ }^{9}$ Another study by Rao et al. showed that mean DTB time for patients was reduced from 90.5 to 60.2 minutes when ECG was transmitted prehospital. ${ }^{8}$ In both studies, prehospital CVL activation was done if STEMI was diagnosed on the transmitted ECG prior to hospital arrival.

As such capabilities are only available to patients arriving by emergency ambulance, this might account for some of the reduction in DTB time that our study has identified. The main timings impacted by mode of transport relate to processes within the ED such as obtaining an ECG, with no impact on timings after CVL activation. This suggests that there are workflows within the ED pertaining to the patients arriving via emergency ambulance that allow for speedier CVL activation, and deeper analysis of the processes might identify potential areas of improvement for patients arriving via own transport. Notably, a prior study by Lee et al. has suggested that door-to-ECG time is a key in reducing DTB time. ${ }^{17}$ In their study, they achieved a median door-to-ECG time of 5 minutes for walk-in patients.

\section{Low rates of emergency ambulance usage}

Our study population had a low proportion of STEMI patients who arrived via emergency ambulance. Compared to an emergency ambulance usage rate of $50-60 \%$ reported by national and international studies, ${ }^{11,12}$ our study population had an ambulance usage rate of $30.8 \%$. This could be due to the National Heart Centre Singapore being co-located with our hospital. Existing patients on follow-up with the specialist centre as well as patients who wish to be treated there might opt to be transported via their own transport, as there is public awareness that emergency ambulances do not allow users to choose their destination, and always convey patients to the nearest hospital. This is an area that would benefit from public education campaigns on the appropriate usage of ambulances.

\section{Other factors affecting DTB time}

When we analysed other factors that impacted various components of DTB time, we noted that presentation after hours was associated with a longer DTB time, which was largely related to longer timings after activation of CVL. This is in agreement with a large prospective observational study in England, which showed that after-hours admission was associated with a longer DTB time. ${ }^{16}$ Our findings are unique as we were able to break down the components of DTB time to see which aspect was impacted. Based on our findings, we recommend that departments looking to improve after-hours DTB times focus on the processes after CVL lab activation such as movement of patients to the CVL as well as the call-back process for on-call CVL personnel. 


\section{Further research}

Three areas would benefit from further research. Firstly, exploring the reasons behind a patient's choice of transport to the hospital would guide the crafting and delivery of public education campaigns. For example, a lack of awareness might have contributed to a patient's decision to call an ambulance, and further research would help us understand the extent of this problem. Secondly, deeper analysis of the PCI process in the hospital might identify the reasons for the slower doorto-ECG time we identified among patients who came via their own transport. Lastly, further studies on the impact of reduced DTB time on long-term outcomes of the patients should be conducted.

\section{Limitations}

This was not a randomised trial, and thus certain factors such as presenting complaint or patient comorbidity could have served as confounders. However, as inclusion in the database required clear-cut ECG changes of STEMI and the presence of symptoms, we believe that this should have a fairly small effect.

Secondly, in our institution, the patient's arrival time (first time stamp) is created upon scanning their identification at the screening area. For patients arriving by ambulance, this is done concurrently by the paramedics while the patient receives care (e.g. ECG, assessment). For these cases, if the ECG time was before the registration time, we recorded an ECG time of 0 minute (ECG on arrival). This might represent a source of bias if the patient had delayed ECG but had an even more delayed registration. We think this is unlikely to present a large bias, as the registration process in our institution is electronic, and unlikely to cause a long delay.

Thirdly, the SCDF ECG database might not be complete, as for some cases, the prehospital ECG might be transmitted prior to identification of the patient. Subsequently, when the patient has been identified and registered in the hospital, this prehospital ECG is not linked retrospectively. As such, some of the cases that were deemed not having a prehospital ECG might have actually had one. We tried to mitigate this by a medical record review as physicians document prehospital ECG interpretation and CVL activation.

Fourthly, we did not have information on the patients' clinical presentations, STEMI types, or long-term follow-up. While the entire cohort had to present with ECG changes and symptoms before being included, patients with very typical symptoms might opt for ambulance use, while others with milder/atypical symptoms might opt for their own transport. More subtle ECG changes might also affect the decision by the provider to activate the CVL. These might be sources of bias. Also, while we did not detect a difference in inpatient mortality, outcome differences might be present on long-term follow-up.

Lastly, while we were able to identify certain aspects of DTB time that was affected by mode of transport and time of arrival, the process of CVL activation is unique to every institution and thus the findings might not be generalisable. However, such a framework for studying the process can still serve as a guidance to other institutions looking to improve their DTB times.

\section{CONCLUSION}

For patients with STEMI undergoing PCI, arriving via emergency ambulance is associated with shorter DTB time. Mechanisms such as prehospital ECG transmission and CVL activation could contribute to this, but processes within the ED also account for part of the reduction in DTB time. Considering that only a third of patients with STEMI arrive via emergency ambulance, public education is sorely needed to increase awareness of STEMI symptoms and to utilise emergency transportation when experiencing those symptoms. Further studies that look into the various processes in detail would be useful to guide measures and workflows to improve DTB time, especially for walk-in patients and patients who present after hours.

\section{REFERENCES}

1. De Luca G, Suryapranata H, Ottervanger JP, et al. Time delay to treatment and mortality in primary angioplasty for acute myocardial infarction: every minute of delay counts. Circulation 2004;109:1223-5.

2. McNamara RL, Wang Y, Herrin J, et al. Effect of door-to-balloon time on mortality in patients with ST-segment elevation myocardial infarction. J Am Coll Cardiol 2006;47:2180-6.

3. Rathore SS, Curtis JP, Chen J, et al. Association of door-to-balloon time and mortality in patients admitted to hospital with ST elevation myocardial infarction: national cohort study. BMJ 2009;338:b1807.

4. Carville S, Harker M, Henderson R, et al, on behalf of the Guideline Development Group. Acute management of myocardial infarction with ST-segment elevation: summary of NICE guidance. BMJ 2013;347:f4006. Erratum in 2021:372:m4946.

5. Amsterdam EA, Wenger NK, Brindis RG, et al. 2014 AHA/ACC Guideline for the management of patients with non-ST-Elevation acute coronary syndromes. J Am Coll Cardiol 2014 64:e139-228.

6. Chen FC, Lin YR, Kung CT, et al. The association between doorto-balloon time of less than 60 minutes and prognosis of patients developing ST segment elevation myocardial infarction and undergoing primary percutaneous coronary intervention. BioMed Res Int 2017;2017:1-6.

7. National Registry of Diseases Office, Singapore. Singapore Myocardial Infarction Registry Annual Report 2007-2018. Available at: https://www.nrdo.gov.sg/publications/ami. Accessed on 20 January 2021. 
8. Rao A, Kardouh Y, Darda S, et al. Impact of the prehospital ECG on door-to-balloon time in ST elevation myocardial infarction. Cathet Cardiovasc Intervent 2010;75:174-8.

9. Ong ME, Wong AS, Seet CM, et al. Nationwide improvement of doorto-balloon times in patients with acute ST-Segment elevation myocardial infarction requiring primary percutaneous coronary intervention with out-of-hospital 12-lead ECG recording and transmission. Ann Emerg Med 2013;1:39-47.

10. Savage ML, Poon KK, Johnston EM, et al. Pre-Hospital ambulance notification and initiation of treatment of ST elevation myocardial infarction is associated with significant reduction in door-to-balloon time for Primary PCI. Heart Lung Circ 2014;23:435-43.

11. So DY, Ha AC, Turek MA, et al. Comparison of mortality patterns in patients with ST-elevation myocardial infarction arriving by emergency medical services versus self-transport (from the Prospective Ottawa Hospital STEMI Registry). Am J Cardiol 2006;97:458-61.

12. Ho AF, Loy EY, Pek PP, et al. Emergency medical services utilization among patients with ST-Segment elevation myocardial infarction:
Observations from the Singapore Myocardial Infarction Registry. Prehosp Emerg Care 2016;20:454-61.

13. Singapore Civil Defence Force. Annual report. Available at: https:// www.scdf.gov.sg/home/about-us/media-room/publications/annualreports. Accessed on 18 December 2020.

14. Emmanuel SC, Tan AT, Tunstall-Pedoe H, et al. The operational research study for the Singapore Myocardial Infarction Register. Singapore Med J 1988;29:446-57.

15. Pilgrim T, Heg D, Tal K, et al. Age- and gender-related disparities in primary percutaneous coronary interventions for acute ST-segment elevation myocardial infarction. PLoS ONE 2015;10:e0137047.

16. Jayawardana S, Salas-Vega S, Cornehl F, et al. The relationship between off-hours admissions for primary percutaneous coronary intervention, door-to-balloon time and mortality for patients with ST-elevation myocardial infarction in England: a registry-based prospective national cohort study. BMJ Qual Saf 2020;29:541-9.

17. Lee CK, Meng SW, Lee MH, et al. The impact of door-toelectrocardiogram time on door-to-balloon time after achieving the guideline-recommended target rate. PLoS ONE 2019;14:e0222019. 